This item was submitted to Loughborough's Research Repository by the author.

Items in Figshare are protected by copyright, with all rights reserved, unless otherwise indicated.

\title{
Energy management system for DC microgrids considering battery degradation
}

\author{
PLEASE CITE THE PUBLISHED VERSION
}

https://doi.org/10.1109/PESGM41954.2020.9281580

PUBLISHER

IEEE

VERSION

AM (Accepted Manuscript)

\section{PUBLISHER STATEMENT}

(c) 2020 IEEE. Personal use of this material is permitted. Permission from IEEE must be obtained for all other uses, in any current or future media, including reprinting/republishing this material for advertising or promotional purposes, creating new collective works, for resale or redistribution to servers or lists, or reuse of any copyrighted component of this work in other works.

\section{LICENCE}

All Rights Reserved

\section{REPOSITORY RECORD}

Li, Fulong, Claudio Cañizares, and Zhengyu Lin. 2020. "Energy Management System for DC Microgrids Considering Battery Degradation”. Loughborough University. https://hdl.handle.net/2134/13619312.v1. 


\section{Energy Management System for DC Microgrids Considering Battery Degradation}

Fulong Li

School of Mechanical, Electrical and

Manufacturing Engineering

Loughborough University

Leicestershire, United Kingdom

f.li@,lboro.ac.uk

\author{
Claudio Cañizares \\ Department of Electrical and \\ Computer Engineering \\ University of Waterloo \\ Waterloo, Ontario, Canada \\ ccanizares@uwaterloo.ca
}

\author{
Zhengyu Lin \\ School of Mechanical, Electrical and \\ Manufacturing Engineering \\ Loughborough University \\ Leicestershire, United Kingdom \\ z.lin@lboro.ac.uk
}

\begin{abstract}
DC microgrids require sources that maintain dc microgrid voltages, with Battery Energy Storage Systems (BESSs) being a good option for this task, given their multiple control alternatives. However, BESS cycling is an issue, and thus, this paper proposes an Energy Management System (EMS) for dc microgrids that considers battery degradation. Therefore, an EMS model is proposed and discussed, demonstrating its application for a practical dc microgrid of a building in Xiamen University. The simulation results show that the proposed EMS, which accounts for BES degradation costs, is an effective tool that avoids frequent battery charging and discharging, while maintaining the required de bus voltage.
\end{abstract}

Index Terms-Battery Energy Storage System (BESS), battery degradation, dc microgrid, Energy Management System (EMS).

\section{INTRODUCTION}

DC microgrids are being considered as an efficient structure for the integration of Renewable Energy Sources (RES), such as solar and wind power, providing more adequate and simpler system controllability than conventional ac microgrids [1]. Thus, dc microgrids do not need to deal with frequency issues, and, although voltage control is required, reactive power management is not necessary. In terms of Energy Management Systems (EMSs), dc microgrids are simpler than ac systems, since there is no need to deal with the reactive power nonlinearities. Furthermore, ac microgrids are three-phase power systems, leading to complex and computationally expensive EMS problems based on linear and nonlinear programming models [2], which is not necessary in dc microgrids, as these are simple two-wire systems.

EMSs are part of the top control levels from a hierarchical control perspective in dc microgrids [3], [4]. A typical dc microgrid and its control scheme is shown in Fig. 1, where the interactions of the generating sources are coordinated by a central controller (CC). Previous research works have focused on the primary and secondary control levels of dc microgrids, as for example in the case of droop controls [5]. On the other hand, various EMS papers have mainly focused on energy regulation at lower control levels. Thus, for example, in [6], dc

This work has received funding from the U.K. EPSRC UKRI Innovation Fellowship scheme under grant No. EP/S001662/2 and the European Union's Horizon 2020 research and innovation programme under grant agreement No.734796. Funding from the Natural Sciences and Engineering Research Council (NSERC) of Canada is also acknowledged.

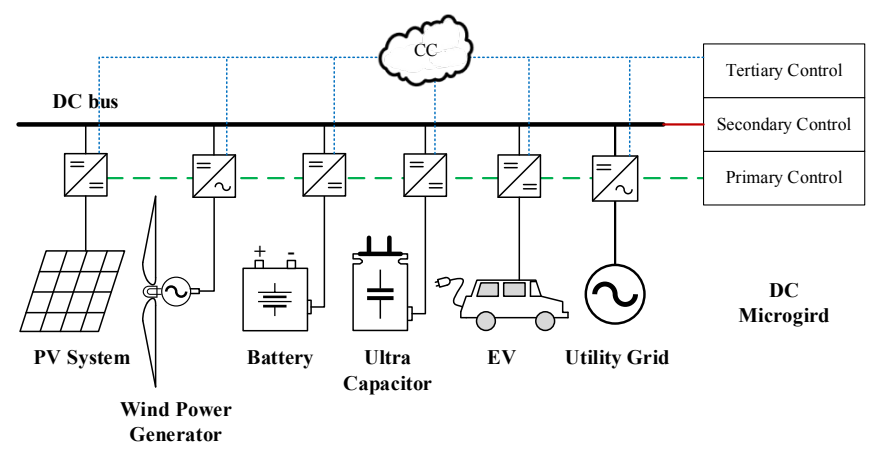

Fig. 1. Typical DC microgrid and its hierarchical control framework.

bus voltage levels are used to indicate the system state, so that energy storage can be regulated through the dc bus, focusing on system dynamic techniques as opposed to optimization approaches for optimal system operation. A rule-based EMS is proposed in [7] for dc microgrid operation, as opposed to applying optimization-based techniques for energy management. To the best of the authors' knowledge, there are not many papers on de microgrid EMS based on optimization techniques similar to those that have been proposed for ac microgrids.

DC microgrids require a generating source to maintain the microgrid bus voltage. Usually, grid-connected bidirectional converters are used for these purposes, since the utility grid has sufficient power to adjust to the dynamic power changes of the microgrid. However, when the microgrid is operated in islanded mode, a local generating source must maintain the system dc bus voltage, using typically Battery Energy Storage Systems (BESSs) for these purposes, given their bidirectional power flow capability, and their ability to regulate voltage through interface converters. In fact, the need for a seamless switch for on-grid and off-grid operating modes can be avoided by using BESS to regulate bus voltages in dc microgrids directly [8]. However, BESSs have limited energy capacity and are affected by their Depth of Discharge (DoD), which determines the number of cycles that the battery can withstand before failure 


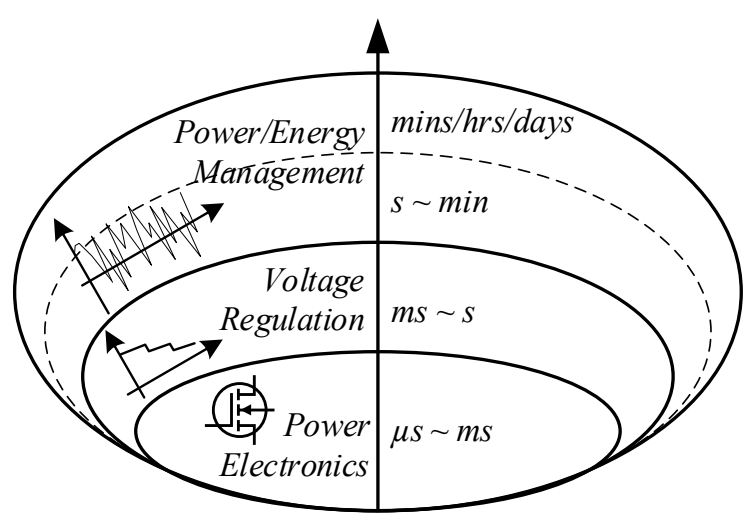

Fig. 2. Hierarchical control scheme of a dc microgrid.

[9]. Therefore, to increase a BESS lifespan, frequent charging and discharging needs to be avoided by maintaining the battery's State of Charge ( $\mathrm{SoC})$ at certain healthy levels, thus minimizing battery degradation.

There are several research works considering battery degradation costs in ac microgrid EMS. Thus, for example, a cooperative distributed energy scheduling algorithm is proposed in [9], studying the impact of DoD on battery lifespan. In [10], the authors present an EMS based on a stochastic dual dynamic programming model that considers battery degradation costs for optimal microgrids operation. The authors in [11], study battery degradation costs compared with utility electricity costs in ac microgrid operation. These and other papers focus on battery degradation in the context of ac microgrid EMS; however, the relevance of battery degradation in dc microgrid EMS has not yet been considered, to the authors' best knowledge.

Based on the aforementioned shortcomings in the existing technical literature, the current paper focuses on an EMS design for $\mathrm{dc}$ microgrids. The battery storage is used to maintain the $\mathrm{dc}$ bus voltage in the microgrid, and a battery degradation model is integrated into an EMS system model, proposing a degradation cost model for the EMS objective function. The proposed EMS model is simulated on a model of an existing grid-connected dc microgrid of a building in Xiamen University. Hence, the main contributions of this paper are the proposed function to represent battery degradation costs in dc microgrids, and an EMS model for optimal operation of BESSbased dc microgrids.

The rest of this paper is organized as follows: Section II reviews the basic control approach for dc microgrids, discussing the possible working modes of these microgrids. Section III describes the proposed EMS and battery degradation models, and a realistic case study and associated simulation results to demonstrate of the application of the proposed EMS are presented and explained in Section IV. Finally, relevant concluding remarks are provided in Section V.

\section{BACKGROUNDS}

\section{A. EMS Overview of DC Microgrids}

The hierarchical control scheme shown in Fig. 2 is normally applied in de microgrids [3]. Primary controls deal with the

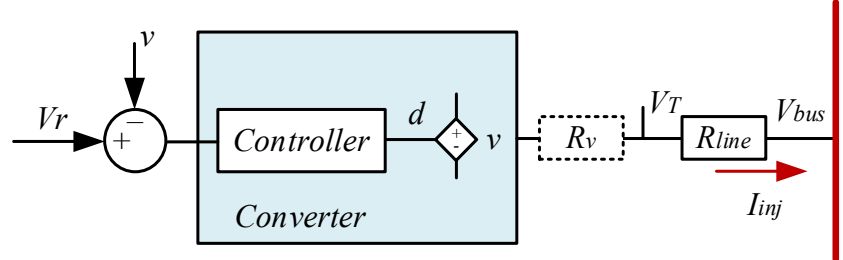

Fig.3. An equivalent model of interface converter based renewable energy sources.

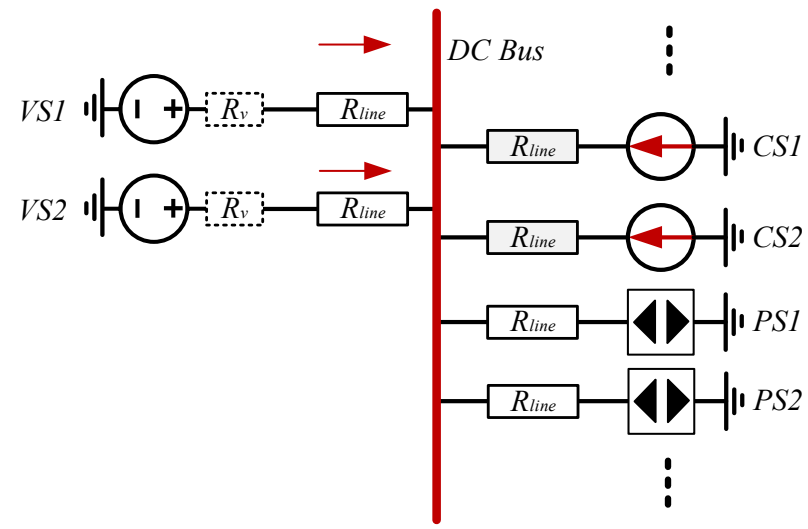

Fig. 4. Simplified model of renewable energy sources in a dc microgrid.

direct control of power electronic devices, and secondary controls are associated with dc bus voltage regulation, typically through droop controls. An EMS would be normally part of a tertiary control level, with either centralized or distributed control approaches, in a time scale of seconds to days. In this context, the EMS aims to schedule dispatchable microgrid generation in the most efficient manner to, for example, minimize running costs and power losses.

\section{B. DC Microgrids Operation}

In dc microgrids, there must be at least one dc voltage source to regulate the bus voltage [8], with the configuration of a microgrid determining how power is dispatched. For instance, if grid interface converters are used to maintain the bus voltage, the grid would take care of any power shortage or surplus from the microgrid. However, when the system is operated in islanded mode, other converters must be assigned to maintain dc bus voltage. In most cases, BESSs are used for these purposes in isolated mode, given its bidirectional power flow capabilities. For grid-connected systems, if a BESS is tasked with constantly maintaining the microgrid dc bus voltage, a seamless switch is not required for proper system operation.

An equivalent control model of a generating source in a dc microgrid is illustrated in Fig. 3, where $V_{r}$ is the controller reference voltage; $V_{T}$ is the converter terminal voltage before considering the line resistance; $V_{b u s}$ is the bus voltage; $I_{i n j}$ is the current injected to the common dc bus from the distributed energy sources; $R_{v}$ is the virtual resistance; and $R_{\text {line }}$ is the line resistance. A simplified model of a dc microgrid is shown in Fig. 4, which illustrates various sources usually encountered in dc microgrids, i.e., voltage sources, current sources, and power sources. If the line resistances are not considered in this dc system, then current sources are equivalent to power sources in terms of energy calculations. For voltage sources, if there is 


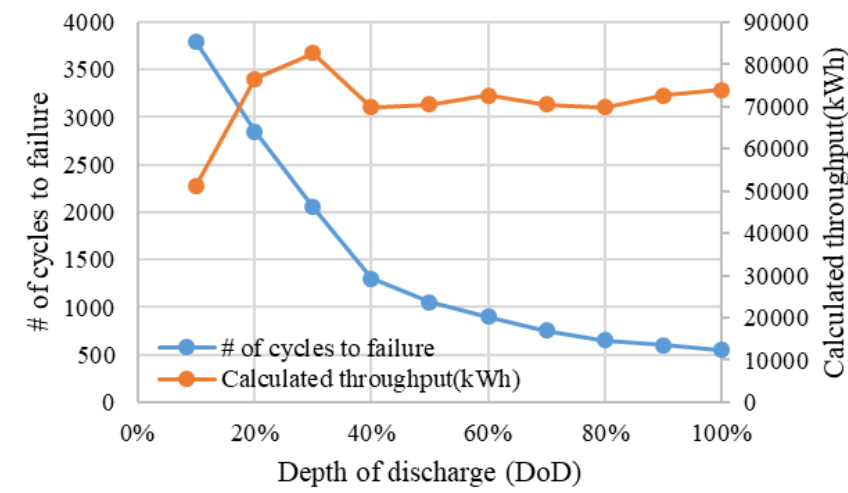

Fig. 5. Cycles to failure and total throughput for a lead-acid battery.

only one in the system, droop control is not required; however, if there is more than one, droop control is needed due to large circulating currents, because the output impedance at low frequencies tends to zero in tightly regulated dc-dc converters. On the other hand, if the line resistances are considered, the power injected from current and power sources is not the same, since for the power source, some of the power is consumed in the line resistance, increasing the terminal voltage. Without droop control for these sources, the system will lack controllability, since the line resistance is non-controllable, as opposed to the converter virtual resistance. For microgrid EMS, line resistances may be neglected, given the small impact that the grid has in a microgrid for dispatch purposes, due to the relatively short length and high amperage of wires, as demonstrated for ac microgrids in [2].

\section{EMS MODELS}

\section{A. Battery Models}

For BESS, the charging/discharging effect on SoC can be represented by the following constraint [12]:

$$
S o C_{b a t, t}=S o C_{b a t, t-1}+\left(\eta_{c} P_{b a t, t-1}^{c}-\frac{P_{b a t, t-1}^{d c}}{\eta_{d}}\right) \Delta t
$$

$$
\operatorname{SoC}_{\text {bat,min }} \leq \operatorname{SoC}_{\text {bat }, t} \leq \text { SoC }_{\text {bat,max }}
$$

where $S o C_{b a t, t}$ is the battery SoC at time $t ; \eta_{c}$ is the charging efficiency; $\eta_{d}$ is the discharging efficiency; $P_{b a t, t-1}^{c}$ is the charged power at time $t-1 ; P_{b a t, t-1}^{d c}$ is the discharged power at time $t-1$; and $\Delta t$ is the time step. The BESS minimum and maximum charging and discharging power constraints at time $t$ can be written as:

$$
\begin{aligned}
& P_{b a t, \text { min }}^{c} \leq P_{\text {bat }, t}^{c} \leq P_{\text {bat,max }}^{c} \\
& P_{\text {bat } \text { min }}^{d c} \leq P_{\text {bat }, t}^{d c} \leq P_{\text {bat,max }}^{d c}
\end{aligned}
$$

Based on the battery degradation model in [9], the degradation cost in $\$ / \mathrm{kWh}$ can be represented as follows:

$$
\varphi=\frac{C_{r p}}{E_{l c}}=\frac{C_{b u} \cdot E_{b}}{2 \cdot \mathcal{L}_{b}(D o D) \cdot E_{b} \cdot D o D}=\frac{C_{b u}}{2 \cdot \mathcal{L}_{b}(D o D) \cdot D o D}
$$

where $C_{r p}$ is the battery's total replacement (capital) cost (assumed here to be $200 \$ / \mathrm{kWh}$ [13]); $E_{l c}$ is the total energy throughput in a lifecycle; $E_{b}$ is installed battery power; $\mathcal{L}_{b}(D o D)$ is a mapping function of battery lifecycles to $D o D$; and $C_{b u}$ is the battery unit cost in $\$ / \mathrm{kWh}$.

The $\mathcal{L}_{b}(D o D)$ for a lead-acid battery in this paper is shown in Fig. 5. Note that large DoDs reduce the cycles to failure, which indicates that over-discharging will reduce the battery lifespan, even though the total throughput would not change. The total battery degradation cost can then be written as:

$$
\begin{aligned}
\mathcal{C}_{d g} & =\sum_{\Delta t} \varphi \cdot\left(P_{b a t, t}^{d c}+P_{b a t, t}^{c}\right) \Delta t \\
& =\sum_{\Delta t} \frac{C_{b u}}{2 \cdot \mathcal{L}_{b}(D o D) \cdot D o D}\left(P_{b a t, t}^{d c}+P_{b a t, t}^{c}\right) \Delta t
\end{aligned}
$$

Observe that to reduce degradation costs, the BESS power usage, i.e., charging and discharging, should be reduced. This limits the BESS contributions to the grid.

\section{B. Operational Constraints}

The battery storage and grid interconnection are both bidirectional but cannot both charge and discharge at the same time. Thus, the following constraints reflect this constraint:

$$
\begin{gathered}
P_{g, t}^{d c} \cdot P_{g, t}^{c}=0 \\
P_{b a t, t}^{d c} \cdot P_{b a t, t}^{c}=0
\end{gathered}
$$

In dc microgrids, there is no reactive power, and the power injected to the grid should be balanced with the power demand. This power balance constraint can be written as:

$$
P_{b a t, t}^{d c}+P_{g, t}^{d c}+P_{P V, t}=P_{E V, t}+P_{A C, t}+P_{L E D, t}+P_{b a t, t}^{c}+P_{g, t}^{c}
$$

where $P_{E V, t}$ is electrical vehicle power; $P_{L E D, t}$ is LED light power.

\section{Objetive Function}

For a grid-connect de microgrid, the following multiobjective function can be defined:

$$
\mathcal{C}_{t}=\min \sum_{\Delta t}\left[\alpha \cdot \xi_{g, t}^{d c} P_{g, t}^{d c}+(1-\alpha) \cdot \varphi\left(P_{b a t, t}^{d c}+P_{b a t, t}^{c}\right)\right] \Delta t
$$

where $\xi_{g, t}^{d c}$ is the local electricity price; $\alpha$ represents a weighting factor, assuming that the battery degradation and grid electricity costs are complementary. This factor can be seen as a competition index of the power throughput between battery storage and utility grid, and thus can be manually adjusted to define the BESS contributions to the dc microgrid.

\section{CASE STUDY}

\section{A. Formulation of the DC System}

The test dc microgrid used here is based on the dc building at Xiamen University [14], which can be represented as in Fig. 6 . This system consists of a set of $150 \mathrm{~kW}$ PV panels; a 200Ah, $336 \mathrm{~V}$ lead-acid battery bank, with its parameters depicted in Table I; and a $160 \mathrm{~kW}$ bidirectional ac-dc converter for grid 


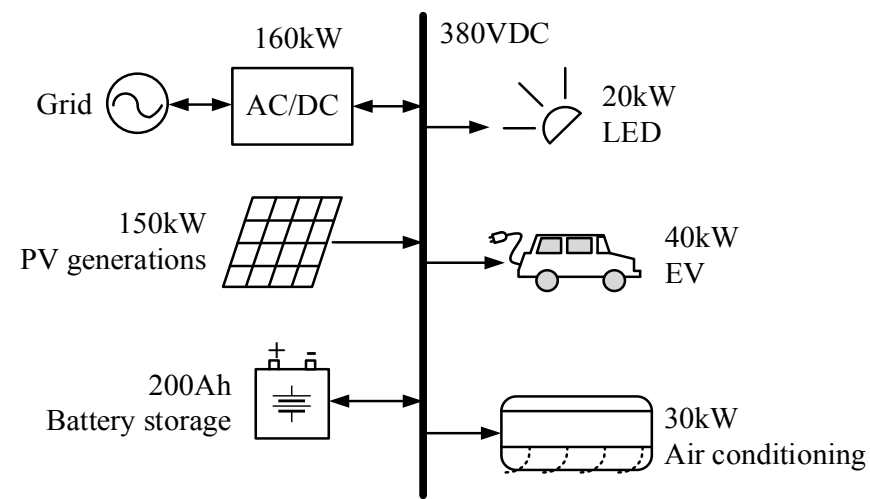

Fig. 6. DC test microgrid.

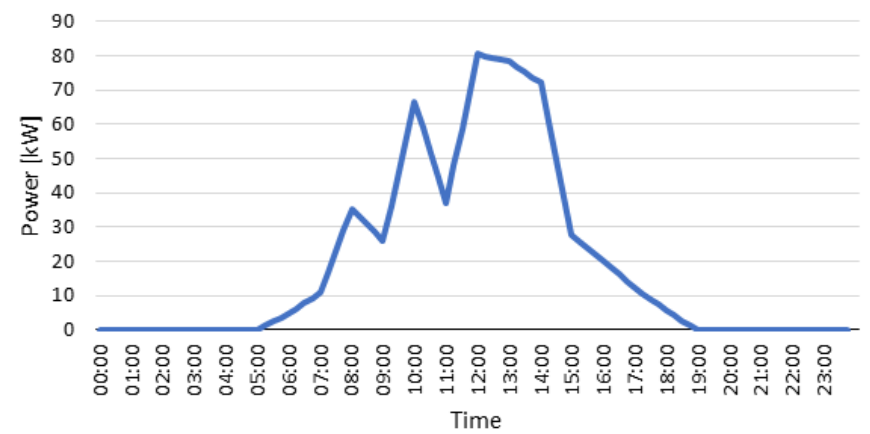

Fig. 7. PV solar generation profile (summer).

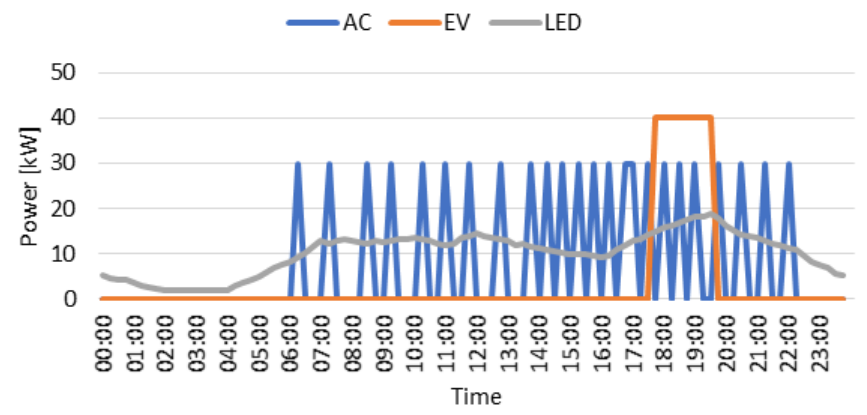

Fig. 8. Daily load profiles.

TABLE I.

BESS PARAMETERS

\begin{tabular}{|c|c|}
\hline Parameter & Value \\
\hline$S o C_{\text {initial }}$ & $80 \%$ \\
\hline$D o D$ & $60 \%$ \\
\hline$S o C_{\text {bat,min }}$ & $1-D o D$ \\
\hline$S o C_{b a t, \text { max }}$ & $95 \%$ \\
\hline$P_{b a t, \text { max }}^{d c}$ & $67.2 \mathrm{~kW}$ \\
\hline$P_{b a t, \text { min }}^{d c}$ & 0 \\
\hline$P_{b a t, \text { max }}^{c}$ & $67.2 \mathrm{~kW}$ \\
\hline$P_{b a t, \text { min }}^{c}$ & 0 \\
\hline$\eta_{c}$ & $95 \%$ \\
\hline$\eta_{d}$ & $90 \%$ \\
\hline
\end{tabular}

connection. On the load side, there are $20 \mathrm{~kW}$ of LED lights, a $40 \mathrm{~kW}$ Electrical Vehicle (EV) charging station, and a $30 \mathrm{~kW}$ Air Conditioning (AC) system. In this system, the battery storage is used to maintain the bus voltage, and line resistances are neglected given the small-scale dc grid, as previously argued.

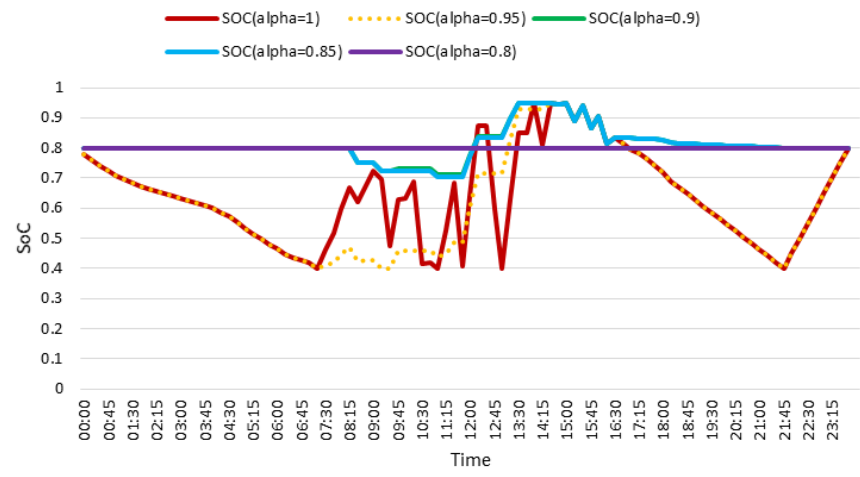

Fig. 9. SoC for different values of $\alpha$.

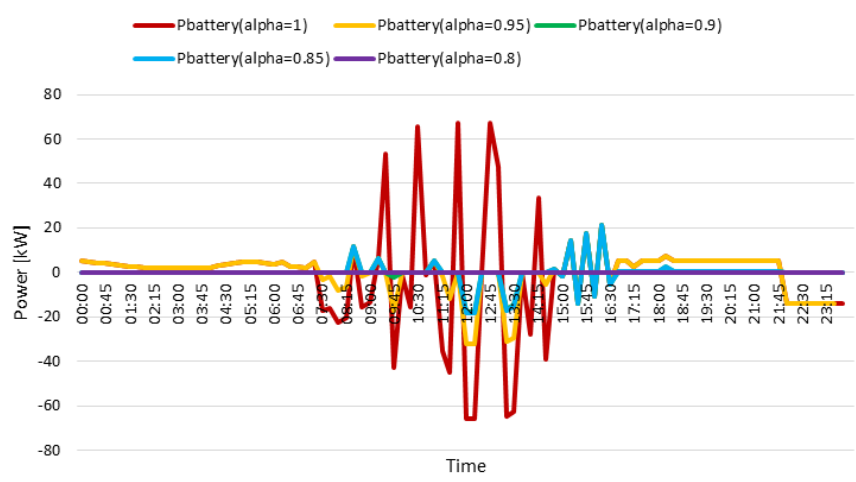

Fig. 10. BESS power for different values of $\alpha$.

The PV generation profile is shown in Fig. 7 [15], and the load profile is illustrated in Fig. 8. The load profiles are depicted in Fig. 8, where the LED load was extracted from [16], the EV charging is assumed to take place during off-work hours, and the air conditioning is an intermittent load operating mostly during work hours. The local daytime electricity price is $0.077 \$ / \mathrm{kWh}$ from $8 \mathrm{am}$ to $10 \mathrm{pm}$, while for the remaining time is $0.044 \$ / \mathrm{kWh}$. The dispatch time interval is assumed to be 15 minutes.

\section{B. Simulation Results}

Applying the optimization model described in Section III, the resulting BESS SoC and power consumption are depicted in Fig. 9 and Fig. 10 for different values of $\alpha$. Observe that when the degradation cost component increases as $\alpha$ decreases, the BESS SoC flattens out, while the charging and discharging power tends to be zero, as expected.

Two representative optimal dispatch solutions are shown in Fig. 11 and Fig. 12. In Fig. 11, note that some of the excess PV power is used to charge the BESS. However, when $\alpha$ decreases, i.e., as the battery degradation cost share increases, this is not the case, resulting in a reduced use of the battery in the dc microgrid, and thus a longer lifespan. 


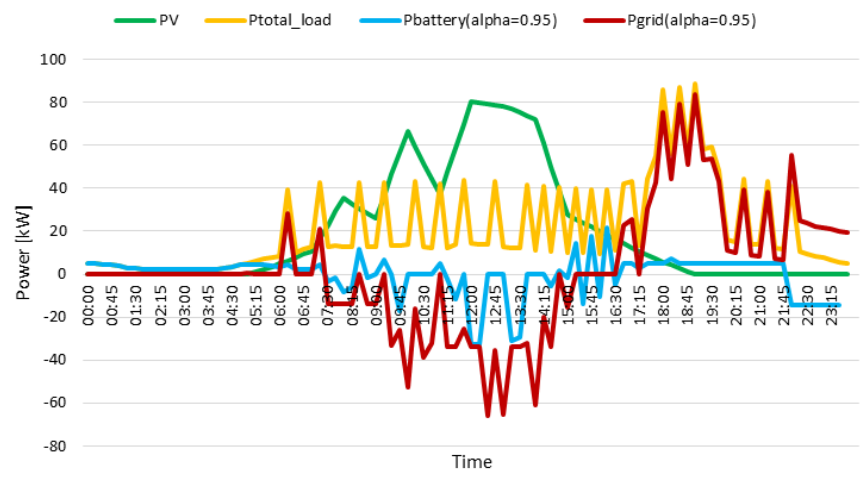

Fig. 11. Optimal model dispatch for $\alpha=0.95$.

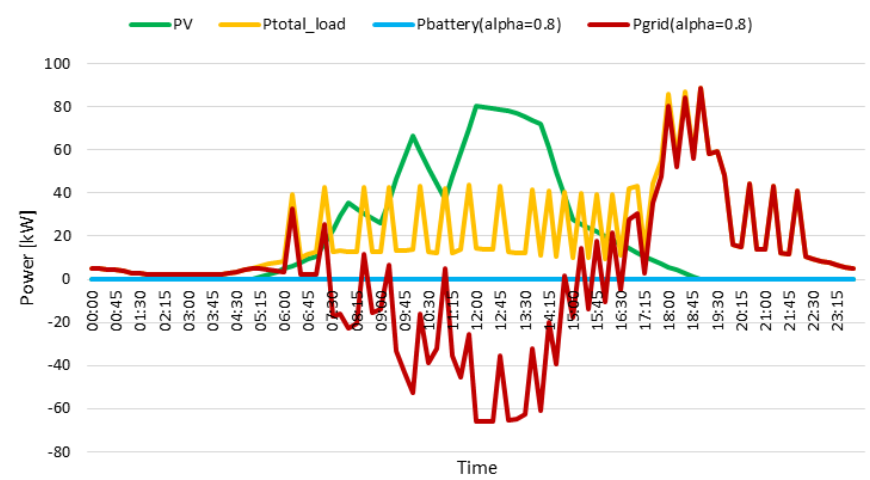

Fig. 12. Optimal model dispatch for $\alpha=0.8$.

\section{CONCLUSIONS}

An EMS model for dc microgrids was proposed in this paper, considering basic control strategies in these types of systems, as well as battery degradation, which reduces the battery storage participation in dc microgrids to increase its the lifespan. The effectiveness of the proposed EMS was demonstrated through simulation results on a practical dc building in Xiamen University, where battery storage maintenance is an issue. The air conditioning and EV loads were modeled as non-dispatchable; however, in future EMS model enhancements, these should be considered as controllable loads.

\section{REFERENCES}

[1] T. Dragicevic, J. C. Vasquez, J. M. Guerrero, and D. Skrlec, "Advanced LVDC Electrical Power Architectures and Microgrids: A step toward a new generation of power distribution networks.," IEEE Electrification Magazine, vol. 2, no. 1, pp. 54-65, March 2014.
[2] B. V. Solanki, C. A. Cañizares, and K. Bhattacharya, "Practical Energy Management Systems for Isolated Microgrids," IEEE Trans.Smart Grid, vol. 10, no. 5, pp. 4762-4775, Sept. 2019.

[3] F. Li, Z. Lin, Z. Qian, J. Wu, and W. Jiang, "A Dual-Window DC Bus Interacting Method for DC Microgrids Hierarchical Control Scheme," IEEE Trans.Sustain. Energy, preprint, pp. 1-10, Jan. 2019.

[4] J. M. Guerrero, J. C. Vasquez, J. Matas, L. G. de Vicuna, and M. Castilla, "Hierarchical Control of Droop-Controlled AC and DC Microgrids-A General Approach Toward Standardization," IEEE Trans.Industrial Electronics, vol. 58, no. 1, pp. 158-172, Jan. 2011.

[5] P. Huang, P. Liu, W. Xiao, and M. S. El Moursi, "A Novel Droop-Based Average Voltage Sharing Control Strategy for DC Microgrids," IEEE Trans.Smart Grid, vol. 6, no. 3, pp. 1096-1106, May 2015.

[6] L. E. Zubieta, "Power management and optimization concept for DC microgrids," in IEEE First International Conference on DC Microgrids (ICDCM), Atlanta, GA, 2015, pp. 81-85.

[7] Y. S. Bhavsar, P. V. Joshi, and S. M. Akolkar, "Energy management in DC microgrid," in International Conference on Energy Systems and Applications, Pune, 2015, pp. 209-213.

[8] Y. Gu, X. Xiang, W. Li, and X. He, "Mode-Adaptive Decentralized Control for Renewable DC Microgrid With Enhanced Reliability and Flexibility," IEEE Trans.Power Electronics, vol. 29, no. 9, pp. 50725080, Sept. 2014.

[9] Yuan Zhang and Mo-Yuen Chow, "Microgrid cooperative distributed energy scheduling (CoDES) considering battery degradation cost," in IEEE 25th International Symposium on Industrial Electronics (ISIE), Santa Clara, CA, 2016, pp. 720-725.

[10] P. Aaslid, M. M. Belsnes, and O. B. Fosso, "Optimal microgrid operation considering battery degradation using stochastic dual dynamic programming," in International Conference on Smart Energy Systems and Technologies (SEST), Porto, Portugal, 2019, pp. 1-6.

[11] Y. Guo, S. Sheng, N. Anglani, and B. Lehman, "Optimal Power Management for Grid-Connected Microgrid Considering Modelling of Different Electricity Cost and Battery Degradation Cost," in 20th Workshop on Control and Modeling for Power Electronics (COMPEL), Toronto, ON, Canada, 2019, pp. 1-7.

[12] D. E. Olivares, C. A. Cañizares, and M. Kazerani, "A Centralized Energy Management System for Isolated Microgrids," IEEE Trans.Smart Grid, vol. 5, no. 4, pp. 1864-1875, July 2014.

[13] "Lead Acid vs LFP cost analysis-Cost Per KWH Battery Storage." [Online]. Available: https://www.powertechsystems.eu/home/techcorner/lithium-ion-vs-lead-acid-cost-analysis/. [Access.: Jun 28, 2019].

[14] F. Zhang et al., "Advantages and challenges of DC microgrid for commercial building a case study from Xiamen university DC microgrid," in 2015 IEEE First International Conference on DC Microgrids (ICDCM), Atlanta, GA, 2015, pp. 355-358.

[15] "PVWatts Calculator." [Online]. Available: https://pvwatts.nrel.gov/pvwatts.php. [Access: Jun 28, 2019].

[16] K. Rudion, A. Orths, Z. A. Styczynski, and K. Strunz, "Design of benchmark of medium voltage distribution network for investigation of DG integration," in IEEE Power Engineering Society General Meeting, Montreal, Que., 2006, pp. 1-6. 